\title{
The effect of nanoparticles on wettability alteration for enhanced oil recovery: micromodel experimental studies and CFD simulation
}

\author{
Peyman Rostami ${ }^{1} \cdot$ Mohammad Sharifi $^{1} \cdot$ Babak Aminshahidy $^{1} \cdot$ Jalal Fahimpour $^{1}$
}

Received: 28 April 2018 / Published online: 4 April 2019

(c) The Author(s) 2019

\begin{abstract}
The applications of nanotechnology in oilfields have attracted the attention of researchers to nanofluid injection as a novel approach for enhanced oil recovery. To better understand the prevailing mechanisms in such new displacement scenarios, micromodel experiments provide powerful tools to visually observe the way that nanoparticles may mobilize the trapped oil. In this work, the effect of silicon oxide nanoparticles on the alteration of wettability of glass micromodels was investigated in both experimental and numerical simulation approaches. The displacement experiments were performed on the original water-wet and imposed oil-wet (after aging in stearic acid $/ n$-heptane solution) glass micromodels. The results of injection of nanofluids into the oil-saturated micromodels were then compared with those of the water injection scenarios. The flooding scenarios in the micromodels were also simulated numerically with the computational fluid dynamics (CFD) method. A good agreement between the experimental and simulation results was observed. An increase of $9 \%$ and $13 \%$ in the oil recovery was obtained by nanofluid flooding in experimental tests and CFD calculations, respectively.
\end{abstract}

Keywords Nanoparticles $\cdot$ Micromodel $\cdot$ Wettability alteration $\cdot$ Oil recovery $\cdot$ CFD calculation

\section{Introduction}

Water flooding is generally one of the most widely used methods for improved oil recovery in oilfields. In such processes, the interactions between rock, oil and injected water on both micro- and macroscales are among the most important parameters controlling the displacement performance. Nanotechnology is also making its entry in all areas of research. The oil and gas industries are picking up on the trend and investing vast amounts of money into new technology to enhance production from existing fields. Possible applications of engineered silica nanoparticles (SNPs) in enhanced oil recovery (EOR) have been investigated, and the literature shows that the unique characteristics of SNPs make them interesting in terms of altering reservoir properties and enhancing oil recovery.

Edited by Yan-Hua Sun

Mohammad Sharifi

m_sharifi@aut.ac.ir

1 Department of Petroleum Engineering, Amirkabir University of Technology, Tehran, Iran
On the microscopic scale, micromodels have been successfully used, helping to visualize the flow patterns during flooding and hence to elucidate the predominant displacement mechanisms (Jamaloei and Kharrat 2010; Buchgraber et al. 2011; Ma et al. 2011, 2012; Maghzi et al. 2011; Kim et al. 2012; Liontas et al. 2013; Conn et al. 2014). The first study of fluid behavior in a micromodel was undertaken by Chatenever and Calhoun Jr (1952). Jamaloei et al. (2016) used micromodels to investigate the effect of wettability on two-phase flow mechanisms and the resulting residual oil saturation. The results from many micromodel flooding studies in the past decades have shown that nanoparticles can improve the performance of injected water to displace the oil (Ju et al. 2006; Cheraghian and Tardasti 2012; Miranda et al. 2012; Hendraningrat et al. 2013; Roustaei et al. 2013). Maghzi et al. (2011) used a five-spot glass micromodel to investigate the chemical injection of nanoparticles in a polyacrylamide (DSNP) solution on oil recovery. Dong et al. (2007) used a micromodel setup to observe the difference between the displacement mechanism in in situ water-in-oil (W/O) emulsion formation and partial wettability alteration. Several core flooding tests were conducted by others using $\mathrm{SiO}_{2}$ nanofluids. Additional oil recoveries of $4.45 \%-10.3 \%$ were observed in their experiments ( $\mathrm{Lu}$ et al. 2017). Silica 
nanoparticles were also used to study foam stability and to analyze the mobility of the stabilized $\mathrm{CO}_{2}$ foam. Foam stability is significantly affected by nanoparticles, and consequently, the oil recovery is increased (Farhadi et al. 2016). Cheraghian (2015) used a five-spot glass micromodel to determine the role of nanoclays in a surfactant-polymer solution as injection fluid in enhanced oil recovery. He found that nanoclay improved the final oil recovery. Li et al. (2017) used a homogeneous glass micromodel to investigate the influence of silica-based nanofluids on displacement mechanisms. They reported that about an extra $25 \%$ incremental oil recovery was recovered by nanofluids compared to deionized water flooding. They reported that silica nanoparticles caused an increase in the oil recovery by altering the wettability to the intermediate-wet condition. The performance of a nanofluid composed of different nanoparticle concentrations in a glass micromodel was also investigated by $\mathrm{Li}$ and Torsæter (2014). Zhang et al. (2018) studied the effect of a polymeric nanofluid in both water-wet and oil-wet fractured micromodels (Zhang et al. 2018). They reported an additional 23.8\% oil recovery using the nanofluid after brine injection in a water-wet system. They concluded that nanofluids are a good candidate for EOR in water-wet porous media and can alter wettability from water-wet to strongly water-wet. The effect of hydrophilic nanoparticles on oil recovery was tested in homogeneous and heterogeneous glass-based micromodels by Suleimanov et al. (2011). They obtained an increase of $17 \%$ and $22 \%$ in oil recovery in homogeneous and heterogeneous models, respectively, compared to the cases where no nanoparticles were used. To visualize the effect of silica nanoparticles as a filtrate reducer on surface rheology, foam slipping and foam stability, a micromodel was also used ( $\mathrm{Lv}$ et al. 2017). They concluded that silica nanoparticles can be used as a high-performance filtrate reducer for a foam fluid. A water-based nanofluid was used to alter the wettability of sandstone rocks from strongly liquid-wetting to intermediate gas-wetting conditions (Gahrooei and Ghazanfari 2017). They measured the contact angles, and their results demonstrated that the SurfaPore M nanofluid has a significant effect on the liquid-phase contact angle. They concluded that the nanofluid is better used in high permeability sandstone rocks. Yu et al. (2015) evaluated the flow enhancement of nanoparticle dispersions in reservoir rocks by conducting different core flood experiments using water-based nanofluids. Their results show the role of the nanoparticle dispersion in controlling the flow on the microscale. Ryoo et al. (2012) investigated the effect of paramagnetic nanoparticles on interface motion by conducting experimental and theoretical studies, developing a magnetic-field-based method for determining the distribution of multiphase fluids in reservoir rocks. They showed that nanoparticles adsorbed at the oil-water and air-water interfaces or dispersed in one of the two fluid phases result in interface displacement. Adsorption of nanoparticles onto minerals is the fundamental issue in choosing the types of nanoparticles. The degree of adsorption would determine the extent of contact angle change and/or decrease in IFT. The role of structural components of disjoining pressure on displacement of the contact line was studied by Wasan and Nikolov (2003). Particle structure formation in the wedge film was confirmed by the theoretical results of Boda et al. (1999).

Pore-scale simulation is a new approach to visualize the effect of microstructure properties on fluid flow behavior in multiphase flow in porous media, and this was performed by several authors. Blunt et al. (2013) presented three pore-scale imaging and modeling studies pertinent to different fields of applications: contaminant transport, carbon dioxide storage in aquifers, and improved oil recovery. They coupled a suite of different numerical techniques with pore space image of rocks on the nanometer scale and presented a methodology to predict flow and transport properties. Afsharpoor et al. (2014) performed several microscale experiments to prove or disprove the hypothesis of viscoelasticity pulling-effect and computational fluid dynamics (CFD) modeling in the dead ends of pores in reservoirs to analyze the flow characteristics of viscoelastic polymers. They concluded that the trapped oil recovery due to the pulling-effect of polymers is not the main factor in reducing the residual oil saturation. Chareyre et al. (2012) proposed three-dimensional pore-scale modeling to effectively solve flow problems with a single fluid phase in a large random polydisperse packing of spheres. They upscaled viscous flow equations at the pore level and approximated with a finite volume numerical scheme. Erriguible et al. (2006) described the transfer between the porous medium and its surroundings using the CFD method. They simulated the convective drying of a piece of a porous medium using CFD software. Gharibshahi et al. (2015) used the CFD method to simulate the effect of pore morphology and its distribution in a 2D micromodel on the enhanced oil recovery factor during nanofluid flooding. They used different homogeneous and heterogeneous patterns in their investigations and concluded that random generation of pore distribution illustrates better results than a homogeneous pore distribution. Modeling and simulation of fluid flow were also studied by others (JoekarNiasar et al. 2012; Icardi et al. 2014).

Looking at the above-mentioned literature, it can be seen that the effects of nanofluid flooding and water flooding were not investigated both experimentally and numerically. More than that and to the best of the authors' knowledge, very few comprehensive studies have been conducted to investigate the effect of nanoparticles on wettability alteration in three cases: water-wet, oil-wet and intermediatewet systems using micromodel flooding. The focus of this work is to study the effect of nanoparticles on alteration of the surface wettability and to compare the effect of different wettabilities in enhanced oil recovery and finally to 
understand the mechanisms dominating the oil entrapment considering the effects of wettability alteration. IFT and contact angle measurements were also taken to investigate important parameters affecting fluid/fluid/rock interaction in static conditions. Then, a glass micromodel device was fabricated to compare the potential of water flooding and silica-based nanofluids on mobilizing the oil phase in dynamic situations. CFD modeling was also used to simulate and to better understand the effect of wettability alteration on the final oil recovery factor. Based on this scope, two series of water flooding and nanofluid flooding tests were conducted in different wettability conditions.

\section{Methodology}

\subsection{Experimental measurements}

\subsubsection{Materials}

Silica nanoparticles used in this study had an average particle size of $20 \mathrm{~nm}$. Two types of aqueous solutions including deionized water and nanofluids (nanoparticles dispersed in deionized water) were used in static and dynamic microscopic tests. The hydrophilic silica nanoparticles were suspended in water; this solution is referred to the silica nanofluid. The nanofluid was prepared with different concentrations: $0.05 \mathrm{wt} \%, 0.1 \mathrm{wt} \%, 0.15 \mathrm{wt} \%$ and $0.2 \mathrm{wt} \%$. The properties of the silica are given in Table 1 . The viscosity and density of nanofluids were measured and were found to be very similar to those of deionized water.

Each solution was mixed using a magnetic stirrer for several minutes. To avoid precipitation of nanoparticles from the solution, an ultrasonic probe $(400 \mathrm{~W}$ and $0.5 \mathrm{~Hz})$ was used to disperse the nanoparticles for $15 \mathrm{~min}$. The $0.2 \mathrm{wt} \%$ nanofluid was placed for 20 days in a closed transparent bottle far from light and heat. After that time, no precipitation or significant color alteration in the sample was detected, showing a good stability of the sample. The properties of the nanofluids of different silica nanoparticle concentrations are listed in Table 2.

It can be seen that although the density and viscosity of nanofluids increase with an increase in the nanoparticle concentration, and this change is not significant.

Table 1 Properties of the silica nanoparticles

\begin{tabular}{lllll}
\hline Particle & Purity & $\begin{array}{l}\text { Average size, } \\
\mathrm{nm}\end{array}$ & $\begin{array}{l}\text { Specific surface } \\
\text { area (SSA), } \\
\mathrm{m}^{2} / \mathrm{g}\end{array}$ & $\begin{array}{l}\text { Bulk density, } \\
\mathrm{g} / \mathrm{cm}^{3}\end{array}$ \\
\hline $\mathrm{SiO}_{2}$ & $>99 \% 20$ & $180-600$ & $<0.10$ \\
\hline
\end{tabular}

Table 2 Silica nanofluid properties

\begin{tabular}{lll}
\hline $\begin{array}{l}\text { Nanoparticle concentration, } \\
\text { wt\% }\end{array}$ & Density at $25^{\circ} \mathrm{C}, \mathrm{g} / \mathrm{cm}^{3}$ & $\begin{array}{l}\text { Viscosity } \\
\text { at } 25^{\circ} \mathrm{C}, \\
\mathrm{mPa} \mathrm{s}\end{array}$ \\
\hline 0.05 & 1.0002 & 1 \\
0.10 & 1.0002 & 1 \\
0.15 & 1.0004 & 1.0002 \\
0.20 & 1.0005 & 1.0002 \\
\hline
\end{tabular}

A crude oil sample obtained from south of Iran was used in experiments, and its physical properties are listed in Table 3.

\subsubsection{Experimental setup}

2.1.2.1 Interfacial tension measurement The interfacial tension (IFT) between crude oil and nanofluids was measured with the pendant drop method. A schematic of the setup is depicted in Fig. 1. The main components of the setup are a backlight, a high-resolution camera, a needle, a low-rate syringe pump and a monitoring system.

2.1.2.2 Contact angle measurement The effect of the silica nanoparticles on the wettability was investigated by measuring the contact angle of the sessile oil droplet on the glass surface immersed in the nanofluid.

Generally, the static measurement is performed by producing a specific drop of oil that is still hanging from a syringe needle, which is then gently brought into contact with the solid. Upon contact with the solid, the drop will detach from the syringe and spread on the surface.

The process of making contact angle measurements begins with capturing a profile image of a sessile drop and an image of an optical scale using the experimental apparatus. The images are processed to extract the drop interface and surface data using ImageJ software.

2.1.2.3 Micromodel and flooding procedure In this work, a micromodel network pattern was designed with the CorelDraw Graphics Suite software and was engraved by melting the glass surface with a laser. The schematic of the porous network is shown in Fig. 2. The micromodel was etched on a nominal 4-mm-thick float plate $4 \mathrm{~cm}$ wide $\times 6 \mathrm{~cm}$ long. The depth of flow channels was $50 \mu \mathrm{m}$, the "grain" size range was

Table 3 Properties of the crude oil sample used

\begin{tabular}{lll}
\hline $\begin{array}{l}\text { Density at } 25^{\circ} \mathrm{C}, \mathrm{kg} / \\
\mathrm{m}^{3}\end{array}$ & $\begin{array}{l}\text { Viscosity at } 25^{\circ} \mathrm{C}, \\
\mathrm{mPa} \mathrm{s}\end{array}$ & $\begin{array}{l}\text { Total acid number } \\
\text { (TAN), mg KOH/g }\end{array}$ \\
\hline 875 & 17.7 & 0.18 \\
\hline
\end{tabular}


Fig. 1 Schematic of the pendant drop apparatus for IFT measurements

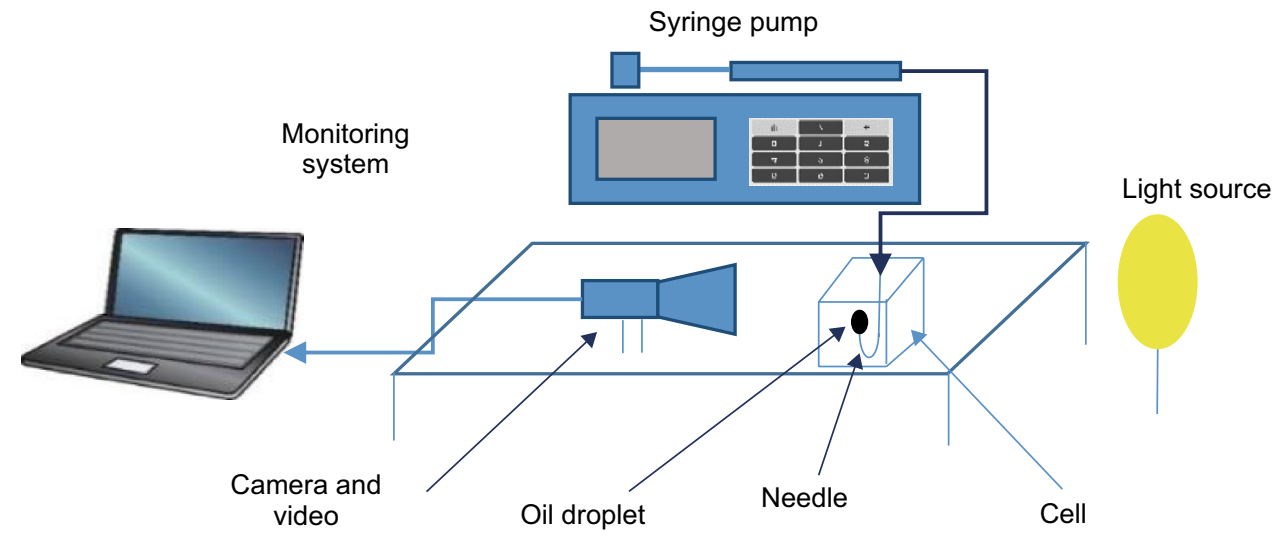

from 1.48 to $2 \mathrm{~mm}$, the porosity was 0.36 , and the average throat size was $200 \mu \mathrm{m}$.

Before each test, the glass micromodel was cleaned with toluene/gasoline and deionized water. The solvents were evaporated in the model with air blowing and applying high temperature. The flooding tests are: (1) water injection into water-wet systems, (2) water injection into oil-wet systems and (3) nanofluid injection into oil-wet systems at a constant rate of $0.04 \mathrm{~mL} / \mathrm{h}$. The displacement procedure in all tests was done in three steps as follows:

1. The micromodel was completely saturated with water.

2. The oil was injected into the micromodel at a constant rate of $0.04 \mathrm{~mL} / \mathrm{h}$.

3. The flooding was conducted with water and nanofluid for two pore volumes (PV) at ambient conditions.

A digital camera was used to capture images and videos during the flooding. The quality of the captured images was improved by image processing software, and the saturations of displacing and displaced phases were determined by ImageJ software. The injection rate in all the stages was $0.04 \mathrm{~mL} / \mathrm{h}$, and the capillary numbers were 0.0047 and 0.0062 for water flooding and nanofluid flooding, respectively. The oil recovery was recorded as the percentage of the initial oil-in-place (IOIP) in different displacement stages.
The basic experimental setup is shown in Fig. 3. A lowrate syringe pump was used to inject a fluid through a plastic tube and to control the flow rate of the fluid through the micromodel. A high-resolution camera with a video recording system was utilized to collect all necessary data. A backlight was also used to improve the quality of the images.

\subsection{Computational fluid dynamic (CFD) modeling}

Following the promising experimental results observed on the positive effect of nanoparticles on oil recovery, a computational fluid dynamic (CFD) exercise was carried out to further improve our understanding of the effect of wettability on fluid displacement. The CFD modeling was performed using Ansys Fluent software. Determining the flow behavior became very faster and easier with Fluent due to parallel computation (Anderson and Wendt 1995; Wendt 2008; Dehkordi et al. 2017). The study of multiphase flow in porous media is a challenging area of fluid mechanics, because both the thermodynamic and non-equilibrium behavior of multiphase systems are not easily described by mathematical models. To work with Fluent, first the geometry of the same 2D heterogeneous network micromodel used during the laboratory tests was generated using SolidWorks ${ }^{\circledR}$ software. The Ansys Fluent software was then used to apply an appropriate gridding scheme to the resulting geometry,

Fig. 2 Schematic of the porous network and a view of pore bodies

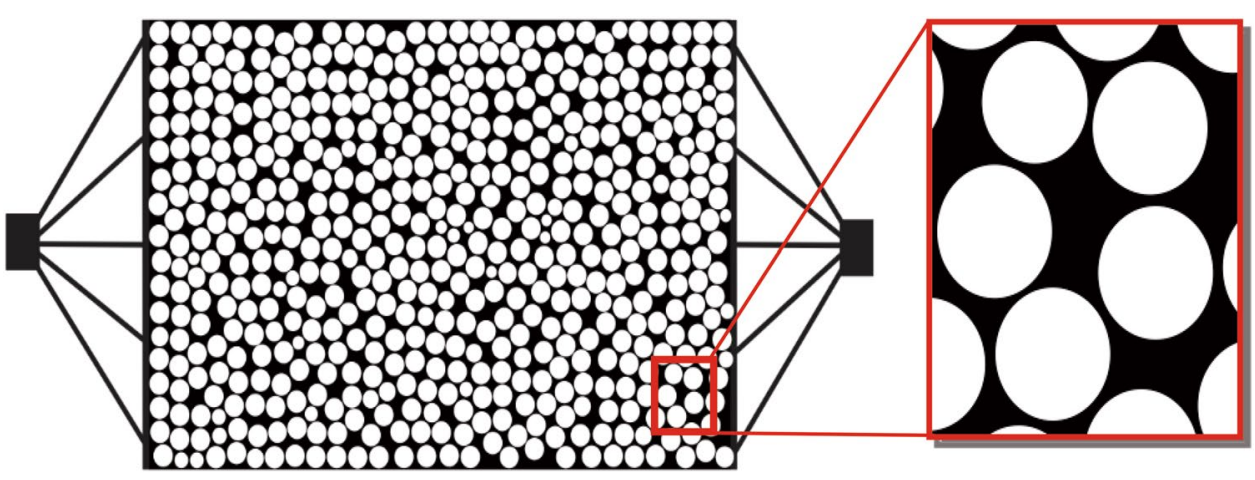


Fig. 3 Schematic of the micromodel setup

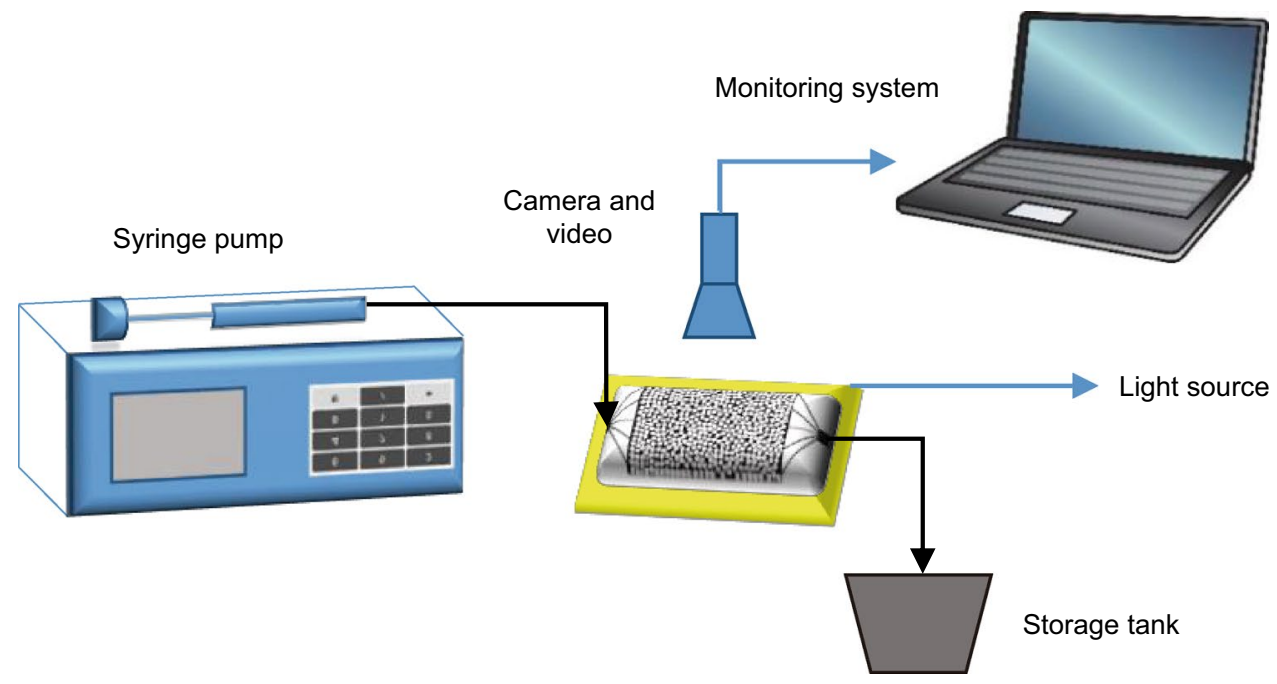

where finally the corresponding momentum and mass transfer equations were solved by employing a finite volume method. The fluid properties were also those corresponding to the fluids used in the micromodel experiment. With this method, the addition of the wall adhesion angle to the volume of fluid (VOF) calculation results was applied in a source term in the momentum equation. Figure 4 shows a magnified section of the meshed model in Ansys Fluent.

The movement of the liquid onto the surfaces is an interesting physical process that has been noticed in many studies. The contact angle becomes an important issue where the surface tension is significant. Focusing on CFD work, three cases of water-wet, oil-wet and intermediate-wet systems were simulated. For this, the simulator allows one to input a static value for the contact angle to adjust the surface normal vector in the cell near the wall. Here, it should be mentioned that the positive effect of nanoparticles on oil displacement has been considered only in terms of the alteration of surface wettability in CFD simulations, according to which the corresponding oil-water contact angles have been changed in the CFD model whenever the effect of nanofluid injection has been analyzed. In all cases, similar to the performed experiments, the model was initially fully saturated with water, and then, the oil injection was started at a constant flow rate of $0.04 \mathrm{~mL} / \mathrm{h}$. During the water injection in all scenarios, two pore volumes were injected into the system. Furthermore, from a series of sensitivities performed on the time steps used for the simulations, it was determined that $\Delta t=0.1 \mathrm{~s}$ can provide accurate results while minimizing the computational costs corresponding to the CFD simulations. Also to assure the accuracy of the final oil recovery obtained, the simulations were continued until the steadystate conditions reached (i.e., no more oil was produced).

\subsubsection{Governing equation}

A volume of fluid (VOF) model as a simplified multiphase model was used to solve the equations. A VOF method can model $n$ phases through solving the momentum, continuity and energy equations. The volume fraction equations for the secondary phases and the algebraic expressions of mass and momentum equations are presented in "Appendix".

To model water flooding and nanofluid flooding using the CFD method, the measured interfacial tension and contact angle from experiments were applied in the VOF model. The surface tension model in Ansys Fluent is the continuum surface force (CSF) model proposed by Brackbill et al. (1992). An option to specify a wall adhesion angle in conjunction with the
Fig. 4 Meshed model in Ansys Fluent mesh window

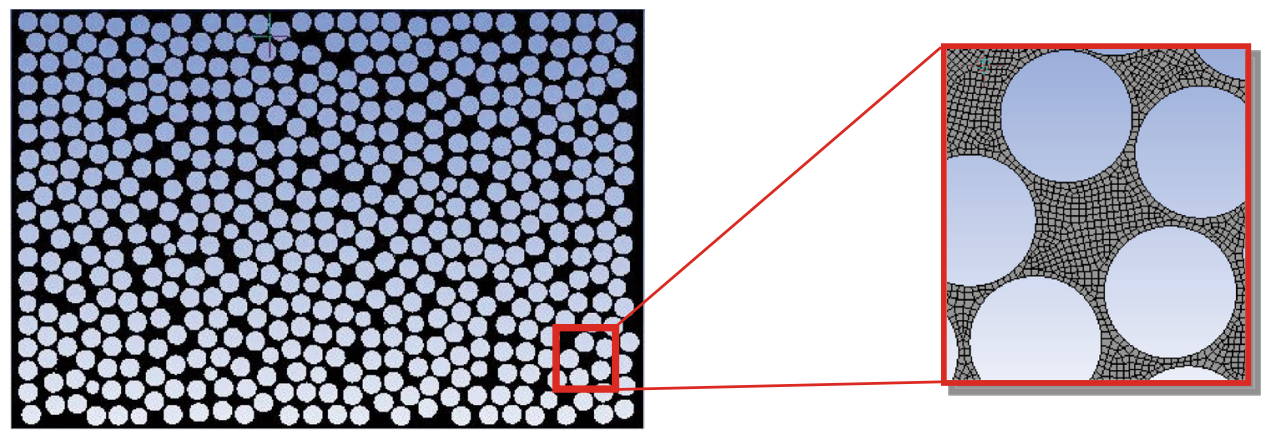


surface tension model is also available in the VOF (Brackbill et al. 1992). This so-called dynamic boundary condition results in the adjustment of the curvature of the surface near the wall.

\subsubsection{Mesh independency study}

To establish the accuracy of the CFD solution, three different mesh sizes were generated to predict the pressure difference between the inlet and the outlet to determine how the mesh quality affects CFD simulation results. The predicted pressure difference between the inlet and the outlet at two difference grid types for all three different mesh sizes was compared. The relative error was used to compare the simulation results for all mesh sizes:

$E_{\mathrm{r}}=\frac{\left|\Delta P_{\text {Finer }}-\Delta P_{\text {Coarser }}\right|}{\Delta P_{\text {Finer }}} \times 100 \%$

where $E_{\mathrm{r}}$ is the relative error and $\Delta P_{\text {Finer }}$ and $\Delta P_{\text {Coarser }}$ are the pressure difference between the inlet and the outlet with finer mesh and coarser mesh, respectively. The mesh size, number of elements and nodes, the value of the relative error and the pressure drop between the inlet and the outlet for each grid type are shown in Table 4. It can be seen that the relative error between two consecutive grids 1 and 2 is low enough and it can be neglected, but the relative error between grid 1 and 3 is greater. Selecting the final grid with larger mesh size and fewer node numbers for the simulation of fluid flooding can help to reduce the number of computation calculations required (Gharibshahi et al. 2015). Therefore, the second type was selected as the main grid for CFD solution to keep the computational costs low.

For the inlet, the inlet velocity and for the outlet, the outlet pressure was used as boundary conditions. For all other faces, the contact angle, in which the fluid is assumed to make with the wall, was used to adjust the surface normal vector to the interface in cells near the wall.

\section{Results and discussion}

\subsection{Interfacial tension}

The measured IFTs between crude oil and aqueous solutions were used as a screening stage to determine the effective range of nanoparticle concentration. Figure 5 shows the measured IFT values and demonstrates that increasing the nanoparticle concentration up to $1 \mathrm{wt} \%$ results in an almost linear decrease in the oil-water IFT, while beyond this value, the effect of increasing nanoparticle concentration on IFT diminishes. Here, it should also be mentioned that all the laboratory tests reported in this study have been conducted at ambient conditions, i.e., $25^{\circ} \mathrm{C}$ and 1 bar.

\subsection{Contact angle}

In this study, the effect of wettability on oil recovery was also investigated by conducting a series of experiments on the water-wet, oil-wet and intermediate-wet porous media. Since the glass surface due to its chemical composition is considered to be water-wet, its original wettability was modified toward oil-wet, for the purpose of experiment performed in this study, using a mixture of stearic acid $/ n$ heptane. According to this, the glass surface was aged by 0.018 molarity of stearic acid $/ n$-heptane solution at $25^{\circ} \mathrm{C}$ for 3 days (Fig. 6). Then, to achieve intermediate-wet surface, the oil-wet glass (Fig. 7) was contacted with the nanofluid for $2 \mathrm{~h}$. Figure 8 shows the intermediate-wet conditions achieved on the glass surface after its contact with the nanofluid.

It should be noted that the sessile drop technique was used to measure the contact angles corresponding to different wettability conditions. Furthermore, to obtain reliable results and to evaluate the repeatability of the experiments

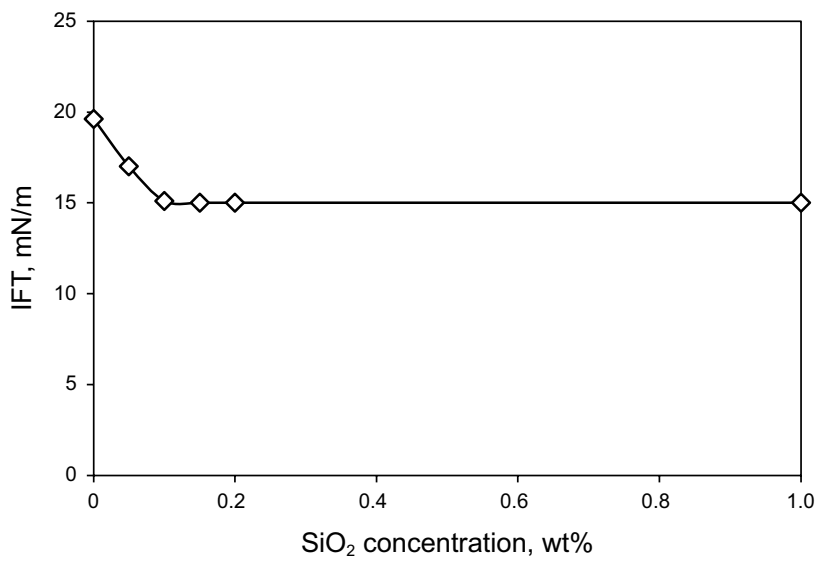

Fig. 5 Interfacial tension of the oil/nanofluid system
Table 4 Mesh independency result for the pressure drop between the input and the output

\begin{tabular}{lllllc}
\hline Grid no. & Mesh size, $\mathrm{m}$ & $\begin{array}{l}\text { Number of } \\
\text { elements }\end{array}$ & Number of nodes & $\Delta P, \mathrm{~Pa}$ & Relative error, \% \\
\hline 1 & 0.0001 & 99,442 & 227,718 & 55.44 & - \\
2 & 0.0005 & 46,773 & 121,365 & 53.32 & 3.82 \\
3 & 0.0010 & 12,652 & 24,561 & 47.65 & 14.05 \\
\hline
\end{tabular}


performed, the contact angle measurements were conducted three times under the same conditions. Therefore, the reported contact angles here are the average of all three

Fig. 6 Close-up views of an oil droplet on the glass surface before (a) and after (b) being aged with stearic acid

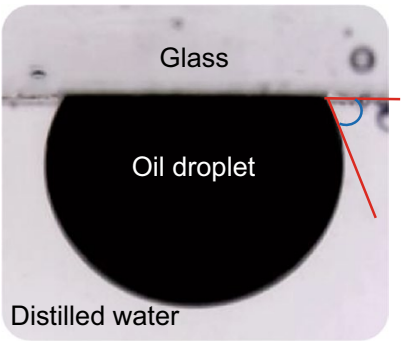

(a) separate measurements. The images of the oil droplet profile on the oil-wet glass surface, before and after $(2 \mathrm{~h})$ being contacted with the nanofluid, are shown in Figs. 7 and 8,
Fig. 7 Close-up views of contact angle prior to being aged in the nanofluid (oil-wet glass)

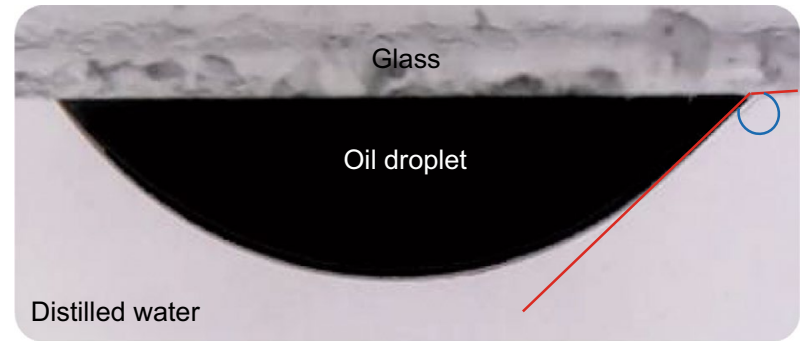

(b)
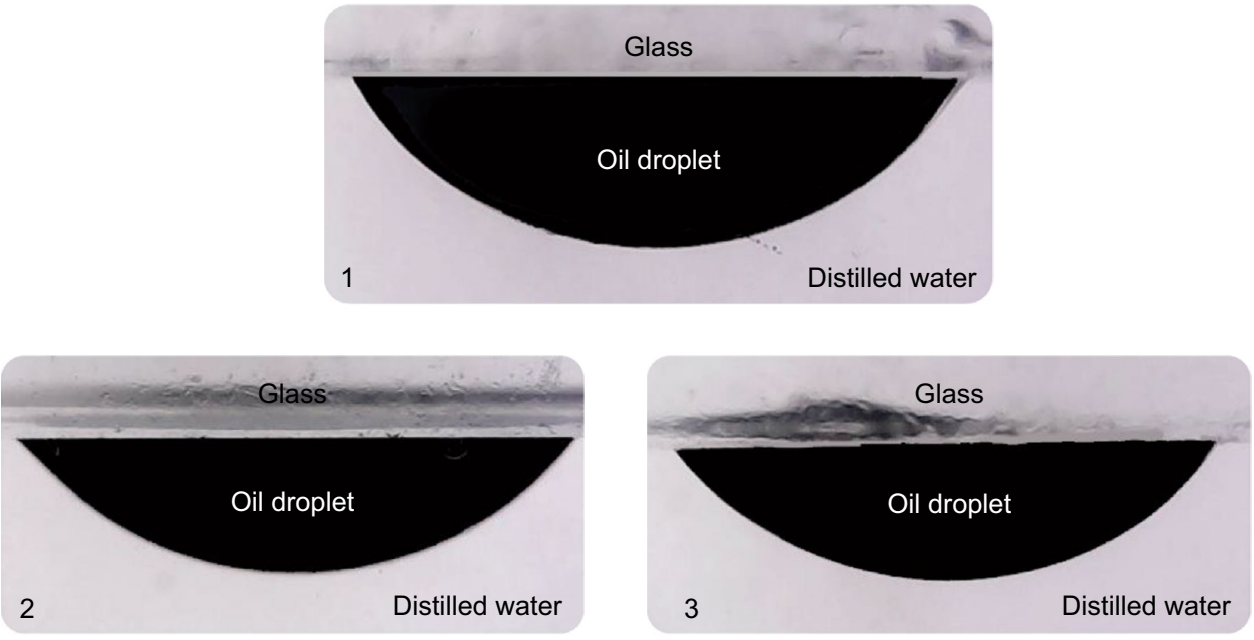

Fig. 8 Close-up views of contact angle after being aged in the nanofluid
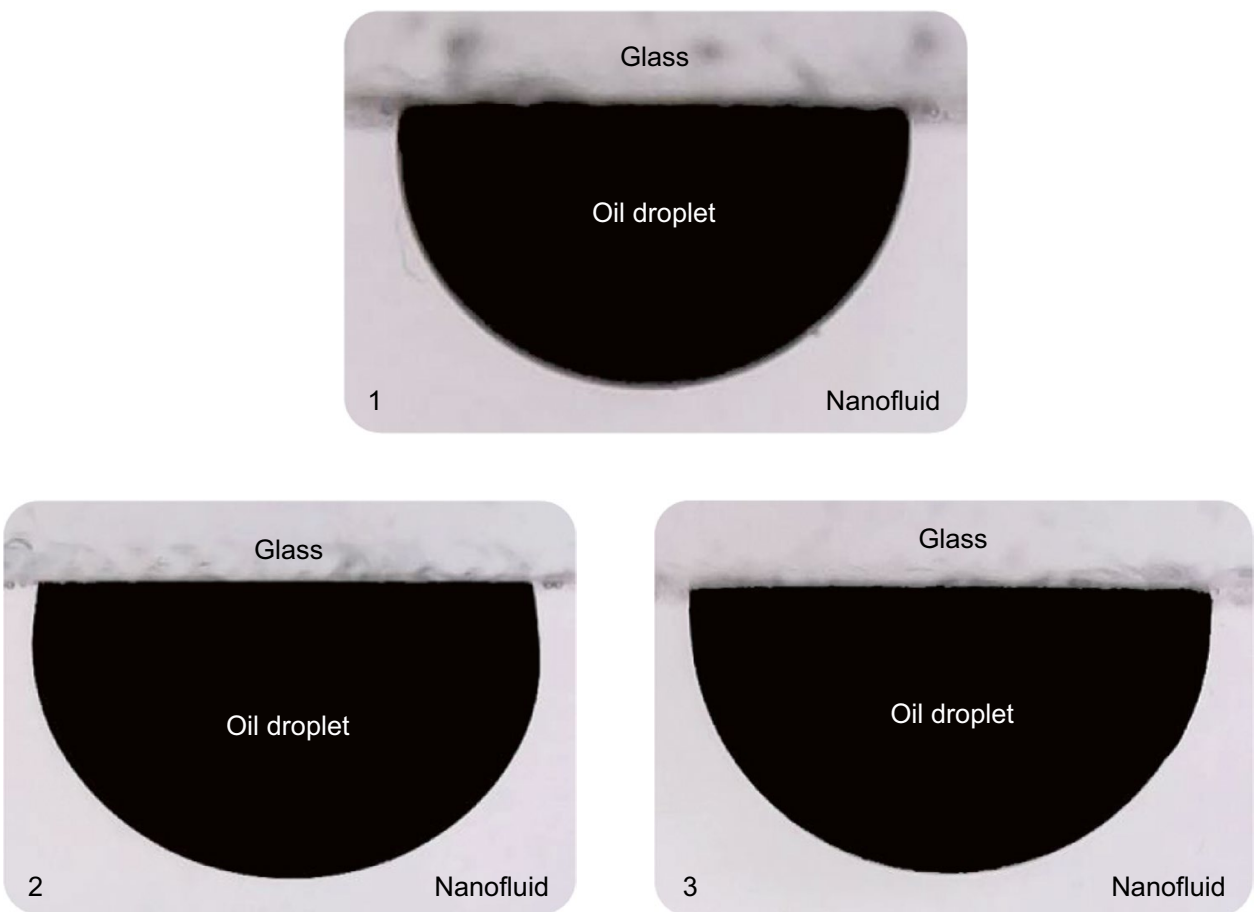
Table 5 Contact angle values after $2 \mathrm{~h}$ of being aged in the nanofluid

\begin{tabular}{lll}
\hline Glass piece & \multicolumn{2}{l}{ Contact angle, degrees } \\
\cline { 2 - 3 } & Before aging & After aging \\
\hline 1 & 135 & 88 \\
2 & 134 & 86 \\
3 & 137 & 93 \\
\hline
\end{tabular}

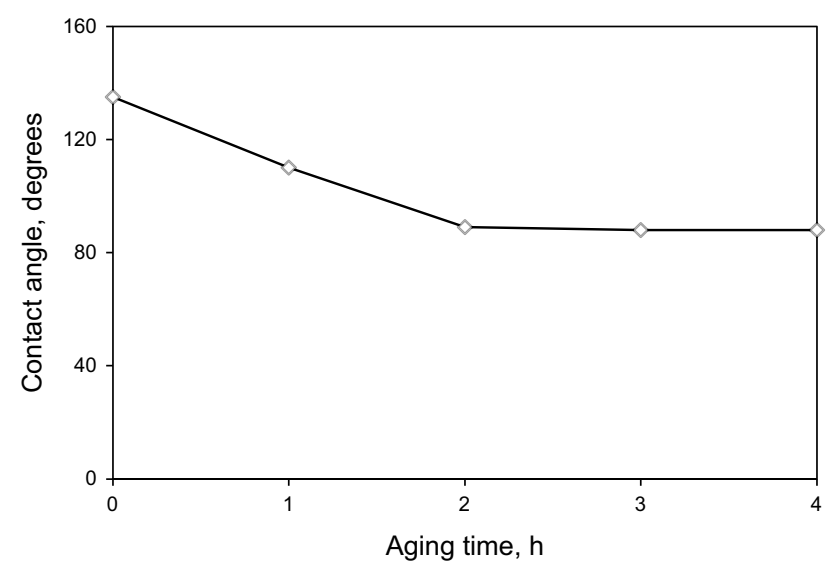

Fig. 9 Average value of contact angles after being aged in the nanofluid for different times

respectively. Table 5 shows the final values of contact angles corresponding to the oil-wet and intermediate-wet glass surfaces. The variation of the contact angles versus time for the oil-wet glass surface immersed in the nanofluid is also depicted in Fig. 9. It is observed that a stable intermediate-wetting state has been achieved after aging in the nanofluid for $2 \mathrm{~h}$.

\subsection{Flooding}

\subsubsection{Water flooding}

The water flooding tests were conducted in water-wet and oil-wet micromodels. Experimental results showed higher oil recovery for the water-wet system compared to that for the oil-wet system. The schematic of the micromodel before and after water injection is shown in Fig. 10.

As Fig. 10 shows, in the water-wet case, water moves in the form of a thin film on the walls of pores and creates a continuous and more stable front, pushing much of the oil out of the pores. This film is shown in the pore-scale view of Fig. 10b which causes the oil to move on the pore walls easily. This film does not appear in the oil-wet model (Fig. 10c). However, in the oil-wet case, water moves in the form of a ganglion through the middle of the larger pores, causing a larger fraction of the oil to remain behind the front. Much of this oil is trapped and cannot be extracted without modifying the chemical makeup of the subsurface fluids. The corresponding trapped oil saturation to the water-wet and oil-wet models obtained from graphical analyses are 0.28 and 0.49 , respectively. Figure 11 shows a pore-scale view of the oil phase entrapped in the water-wet and oil-wet micromodels. It is noticed that the amount of the oil trapped in the latter case is larger.
Fig. 10 Schematic of the micromodel. a Before water flooding. b Water-wet model after water flooding. c Oil-wet model after water flooding

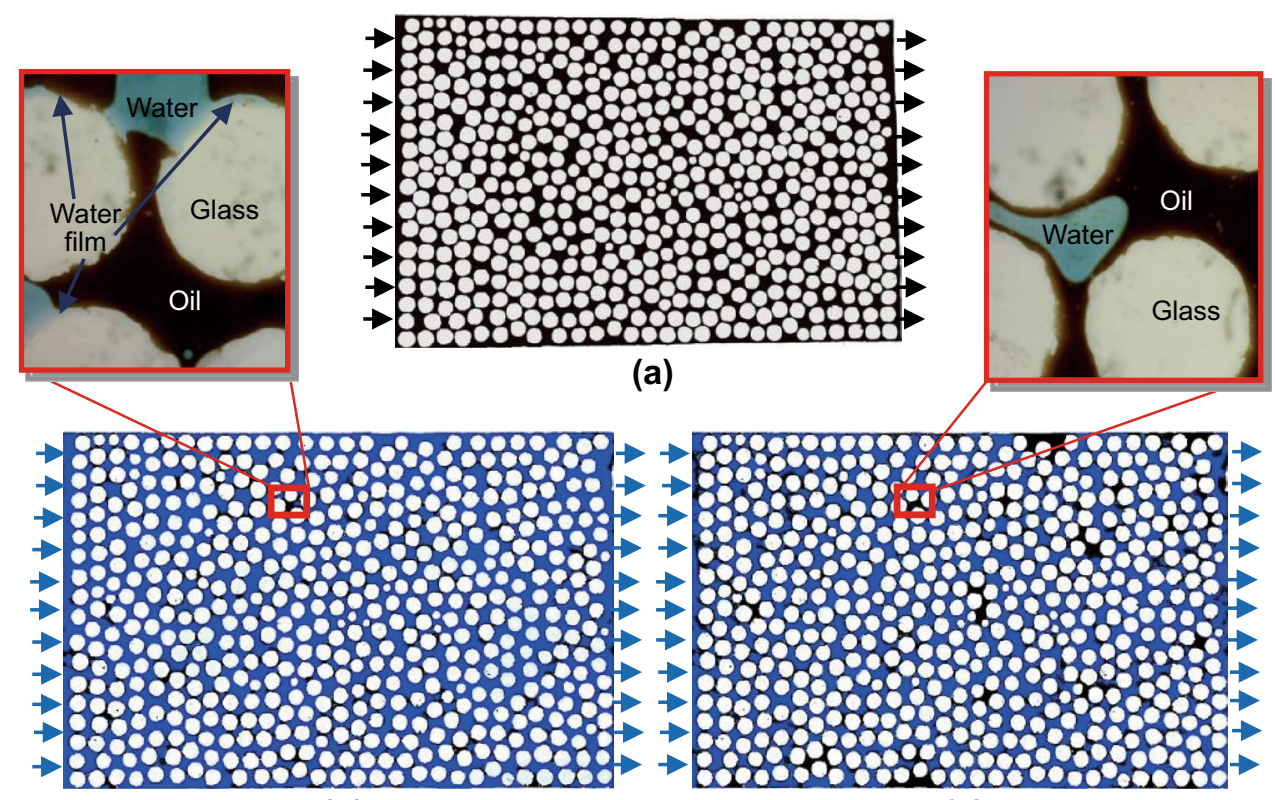

(b) (c) 


\subsubsection{Nanofluid flooding}

The objective of this section is to investigate the effect of $\mathrm{SiO}_{2}$ nanoparticles on surface wettability alteration during nanofluid injection and hence its impact on improved oil recovery. Here, it should be mentioned that the $\mathrm{SiO}_{2}$ nanoparticles were able to alter the wettability of the oil-wet glass surface to intermediate-wet, as demonstrated before through the contact angle measurements discussed in Sect. 3.1.

From the results of IFT measurements (shown in Fig. 5), the nanofluid with a $\mathrm{SiO}_{2}$ nanoparticle concentration of $0.1 \mathrm{wt} \%$ was used. To synthesize the nanofluid, the solution was initially homogenized for $5 \mathrm{~h}$ using a magnetic stirrer, and then, it was ultrasonicated to achieve a more stable dispersion of nanoparticles. The final nanofluid was injected into the water-wet and oil-wet micromodels at a constant rate of $0.04 \mathrm{~mL} / \mathrm{h}$. The corresponding trapped oil saturations to nanofluid injection were reduced by $9 \%$ and $30 \%$ in the cases of water flooding in the water-wet and oil-wet models, respectively, compared to those obtained before for water flooding cases. These differences were related to the positive effect of nanoparticles on alteration of the surface wettability in the flooding process. The displacement profiles of the nanofluid injection and the comparison of oil recovery in different wettability conditions are shown in Figs. 12 and 13 , respectively. It can be seen that the oil recovery in the intermediate-wet system was even larger compared to the water-wet system ( $81 \%$ in the case of nanofluid flooding). It can be hypothesized that in the intermediate-wet systems owing to the reduced effect of capillary forces, the injected fluid has a more piston-like displacement, hence resulting in higher oil recovery.

Figure 14 shows pore-scale views of the nanofluid injection at different time steps. It can be seen that the oil droplets became detached from the pore walls as the contact time between the nanofluid and the glass micromodel increased.

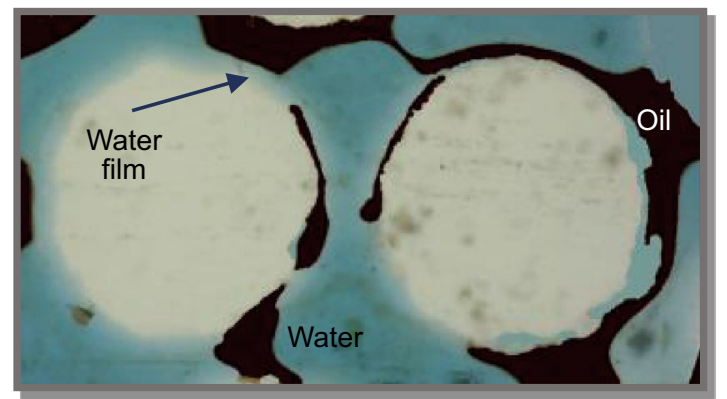

(a)

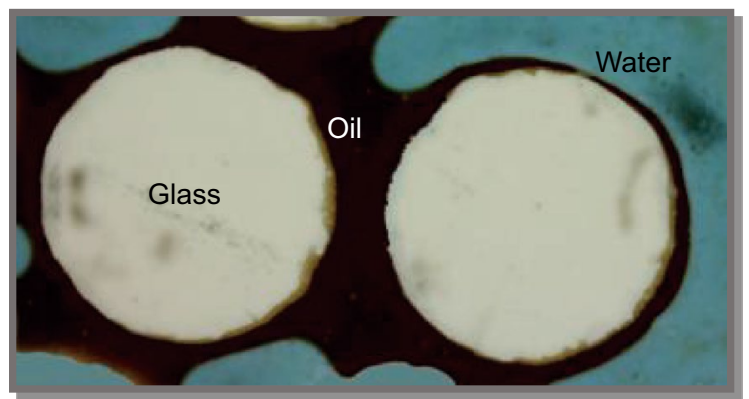

(b)

Fig. 11 Pore-scale views of the distributions of oil in water-wet (a) and oil-wet (b) micromodels

Fig. 12 Oil displacement during nanofluid flooding in the oil-wet system after $1000(\mathbf{a}), 2000$ (b), 3500 (c) 5000 (d) seconds of injection

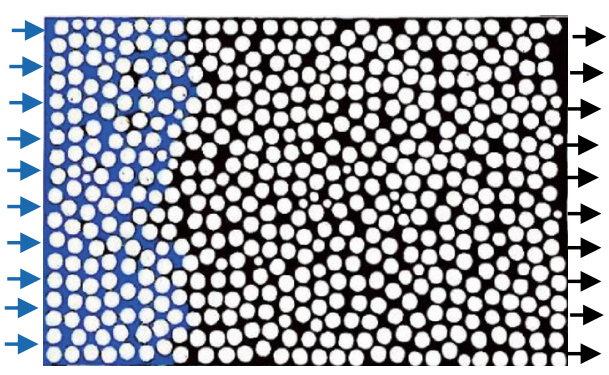

(a)

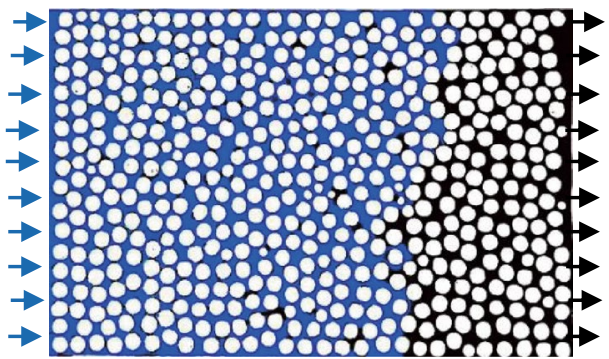

(c)

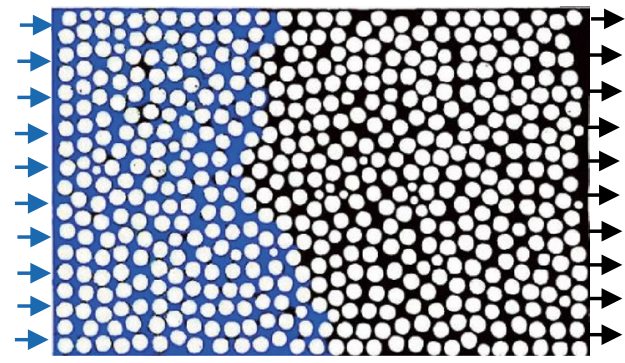

(b)

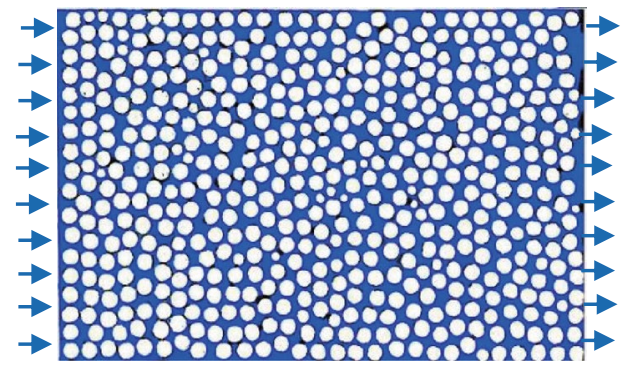

(d) 


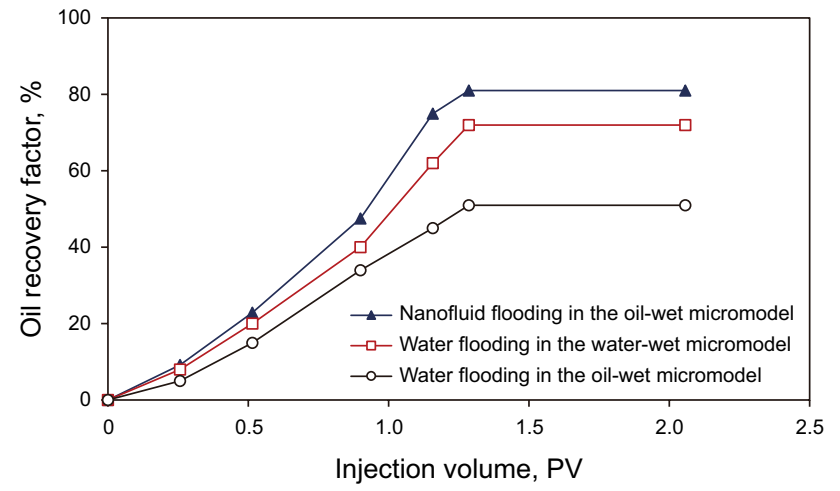

Fig. 13 Oil recovery in different wettability conditions (experimental results)
This observation can be attributed to the formation of a thin film on the glass surface known as the wedge layer (Fig. 15). The subsequent layer imposes a pressure on the discontinuous oil phase, called the disjoining pressure (Moon 2010). By increasing the contact time between the nanoparticles and the glass surface, more nanoparticles are adsorbed on the surface and as a result, the surface wettability changes to the intermediate-wet condition, while the disjoining pressure also increases.

\subsection{The results of CFD simulations}

As mentioned earlier, in CFD simulations, the effect of wettability on oil recovery was assessed by altering the

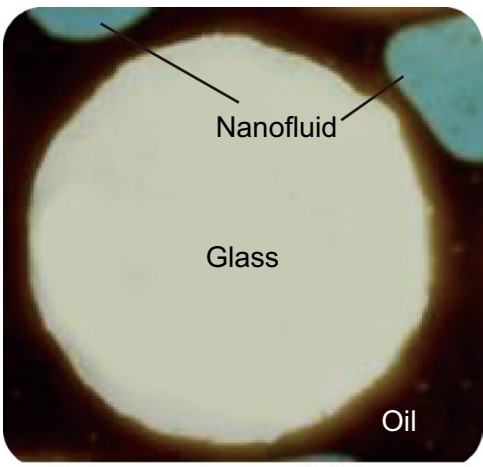

(a)

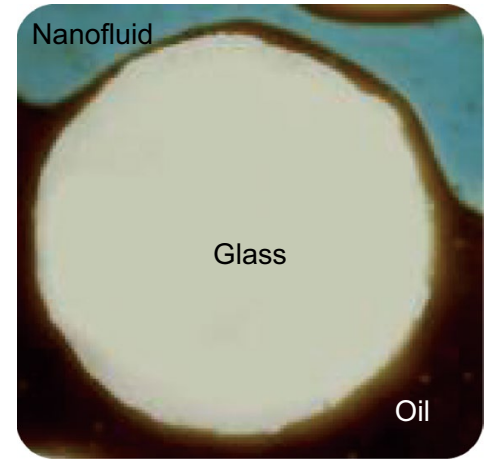

(b)

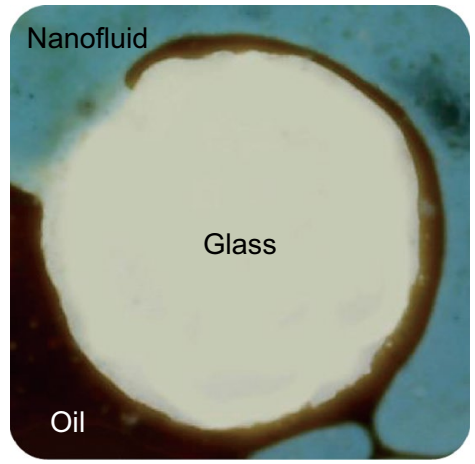

(c)

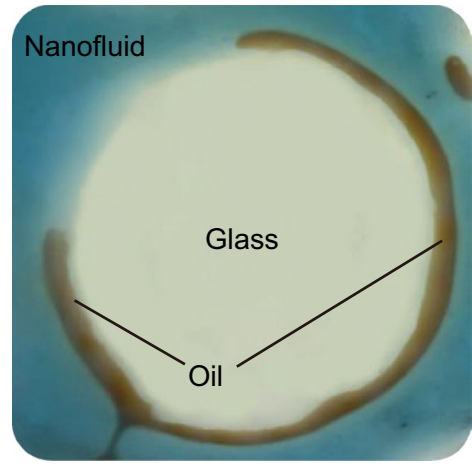

(d)

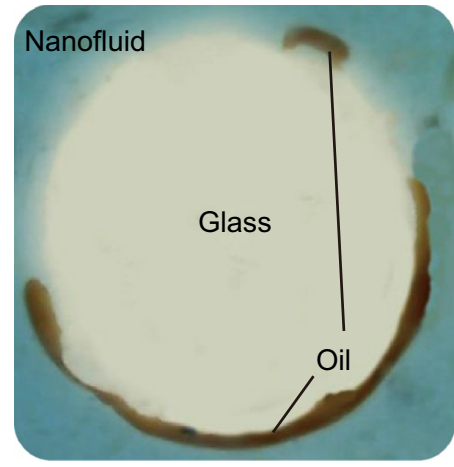

(e)

Fig. 14 Pore-scale views of the micromodel during nanofluid flooding for a period of time: initial time (a), and after 1000 (b), 2000 (c), 3000 (d), 5000 (e) seconds of nanofluid injection

Fig. 15 Illustration of how an oil droplet is loosened from the glass surface by adsorption of silica particles

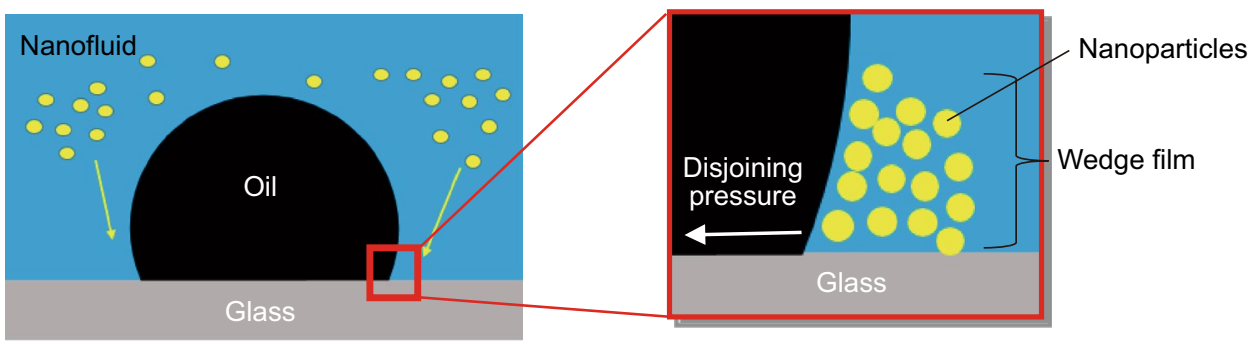


corresponding contact angles of the oil-water system in the network model. In order to do this, three different wettability cases of water-wet, oil-wet and intermediate-wet with the corresponding contact angles of $55^{\circ}, 135^{\circ}$ and $89^{\circ}$ were constructed. In analogy to the micromodel tests discussed before, the first two cases simulate the water injection scenarios in the water-wet and oil-wet media, respectively, while the latter one mimics the nanofluid injection scenario into an oil-wet system, where its final wettability state turns into the intermediate-wet condition owing to the adsorption of nanoparticles. Figures 16 and 17 depict the displacement processes and phase distributions resulting from the CFD simulations for the different wettability cases modeled. Figure 18 also compares the final oil recovery corresponding to these three wettability cases. In line with the experimental results from the micromodels, the intermediate-wet state (nanofluid injection) results in the lowest trapped oil saturation. Table 6 shows the output of the pressure drop in the CFD simulation results.

Table 6 shows that the water-wet micromodel had a lower pressure drop. The intermediate-wet micromodel showed the lowest pressure drop and the oil-wet micromodel showed the maximum pressure drop among all models.

\section{Comparison of experimental and CFD results}

Figure 19 compares the final oil recovery values from the micromodel and CFD studies for different wettability conditions performed in this work. Very good agreement between these two sets of data can be observed. Even in the case of saturation profile comparison as was discussed in the previous section, although we may not see a perfect match between the simulation and experimental results, still both show more stable front movement and less trapped oil in the case of nanofluid flooding compared with water flooding. It is noted that there is a good agreement between the experimental and simulation results, verifying the accuracy of the experiments performed and mechanisms observed during the displacement tests. The oil being trapped depends on different factors such as the structure of pores in the porous medium, fluid-rock interaction and fluid-fluid interaction. In an EOR project, all of the original oil-in-place cannot be recovered, and a considerable amount of oil remains trapped in the porous medium. In this study, it can be concluded that surface wettability is one of the most important factors for recovering oil-in-place. In the oil-wet micromodel after the water flooding, a considerable amount of oil remains trapped in the corners of the pores and the injected water cannot overcome the capillary pressure of the oil phase to mobilize it. However, in the case where nanofluid was injected, the

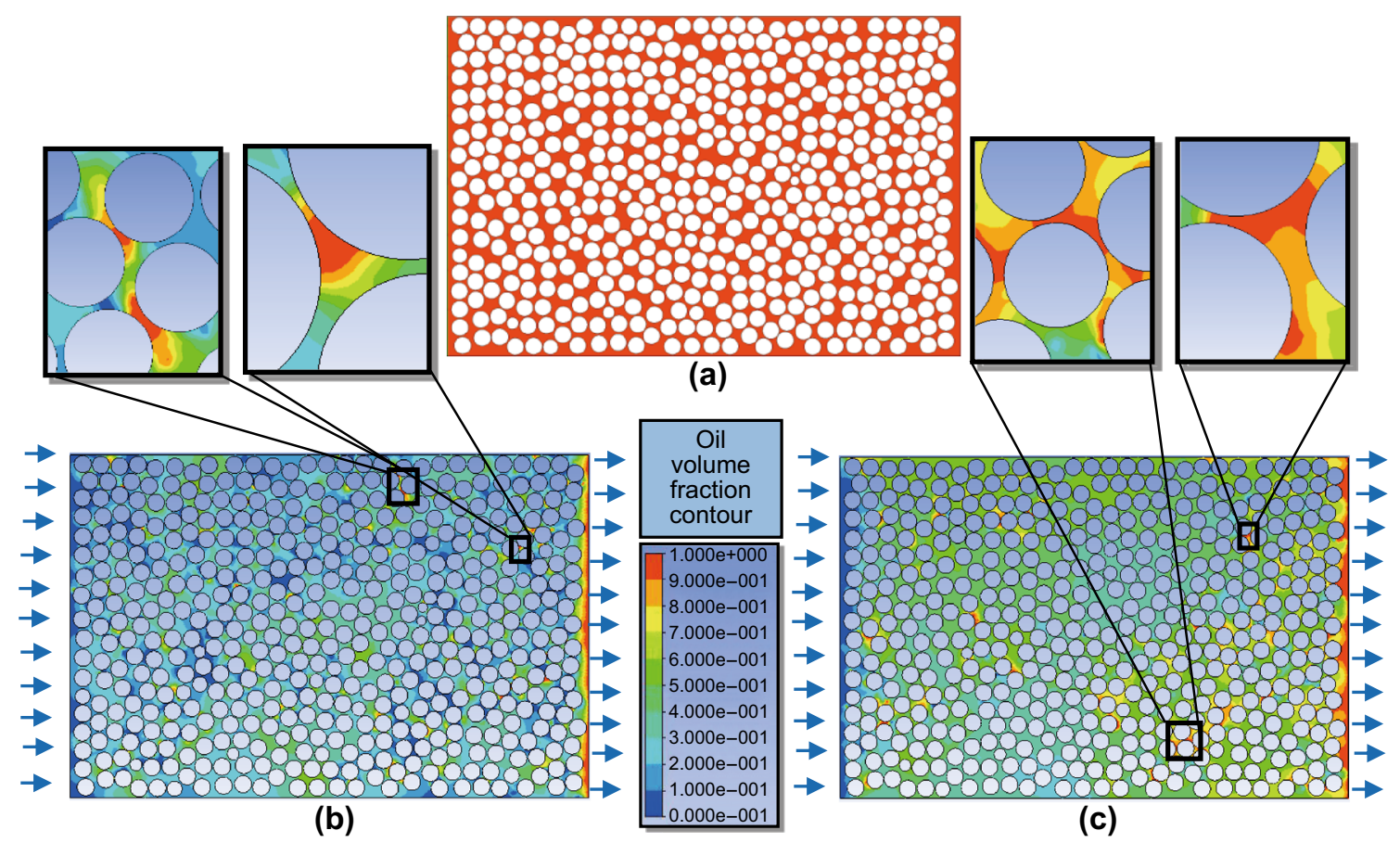

Fig. 16 Schematic of the oil volume fraction contour of micromodels. a Before water flooding. b Water-wet model after water flooding. c Oilwet model after water flooding 


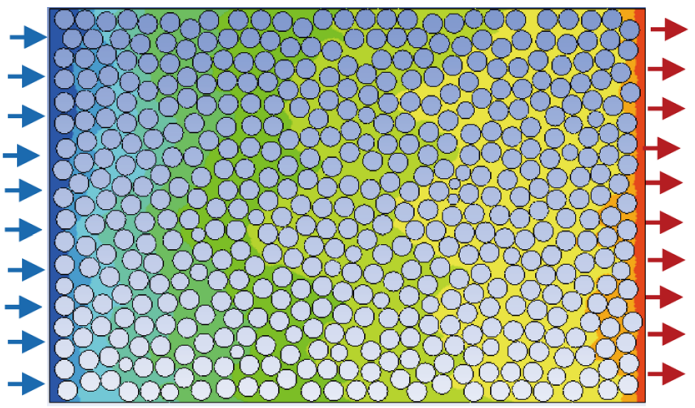

(a)

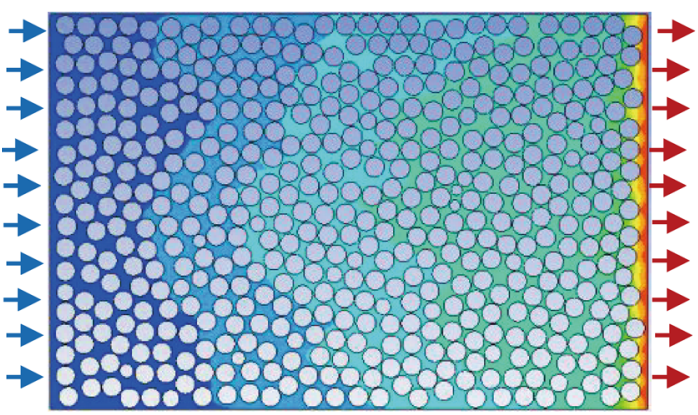

(c)

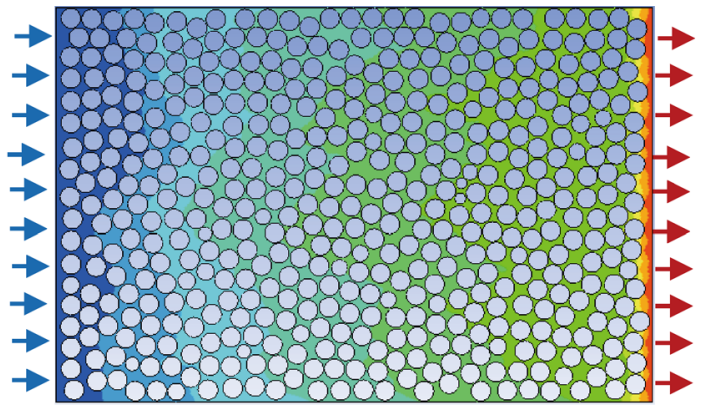

(b)

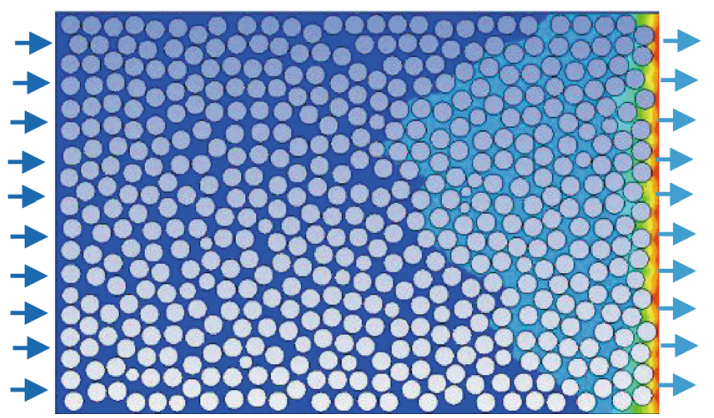

(d)

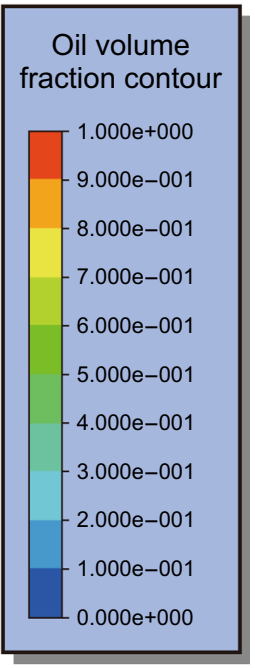

Fig. 17 Contour plots of oil displacement during nanofluid flooding after 1000 (a), 2000 (b), 3000 (c), 5000 (d) seconds of injection

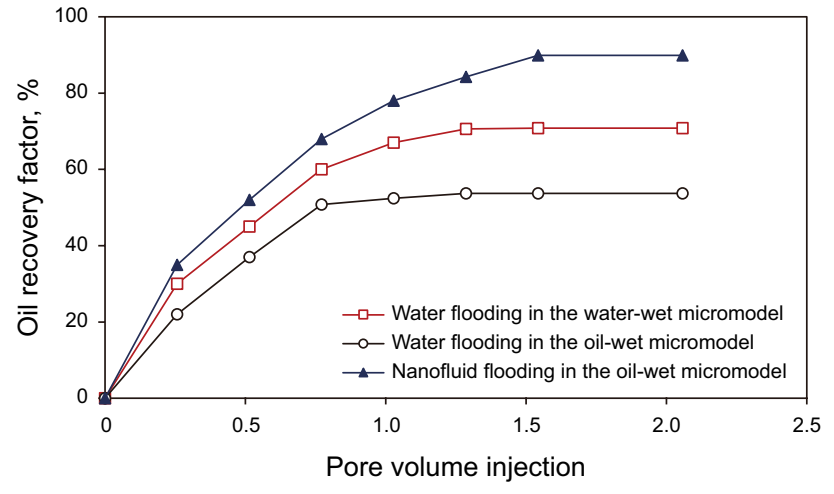

Fig. 18 Schematic diagram of oil recovery in different wettability conditions (simulation results)

Table 6 Pressure drop in the CFD simulation results

\begin{tabular}{lc}
\hline Simulation case & $\begin{array}{l}\text { Pressure } \\
\text { drop, Pa }\end{array}$ \\
\hline Water-wet & 207 \\
Oil-wet & 495 \\
Intermediate-wet & 54 \\
\hline
\end{tabular}

amount of trapped oil decreased considerably. The results from both micromodel experiments and CFD simulations demonstrate that the nanofluid injection, compared to the

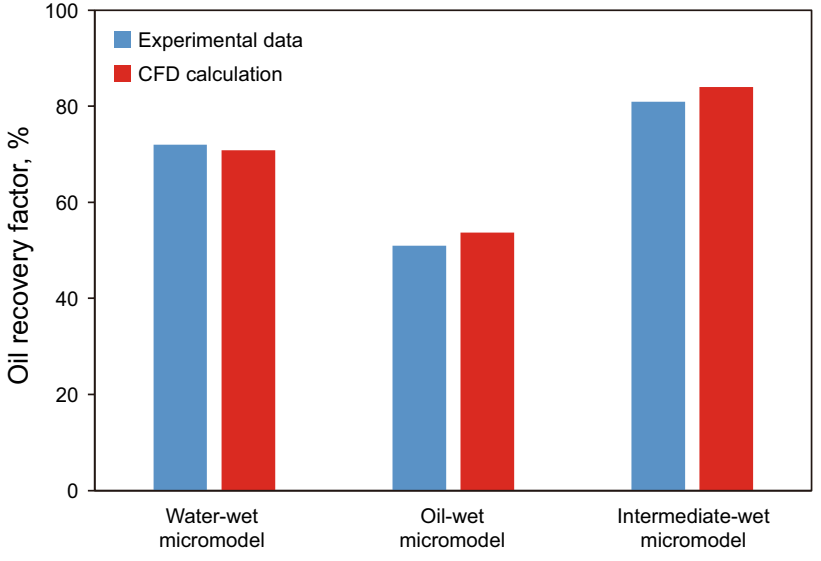

Fig. 19 Comparison of the oil recovery factor obtained from both experimental and CFD results

water injection, provides a more piston-like displacement process; hence, higher oil recovery is expected.

The conducted experiments in the current study show the positive effect of silica nanoparticles in the rock-oil-water system. There is still much investigation to be carried out to obtain a complete overview of how the silica nanoparticles affect fluid properties. The current experimental setup could be modified to higher pressure systems in order to test the oil samples with higher viscosity at a higher injection rate and higher pressure. The measurements can also be performed at 
different temperatures, with other oils and with other nanoparticle concentrations.

\section{Conclusions}

In this study, the effects of silica nanoparticles on the alteration of wettability for improved oil recovery were investigated by performing a series of micromodel experiments and CFD simulations in different wettability conditions. Based on the observed results, the following conclusions can be drawn:

1. The initial wettability of the system is an important factor that affects the trapped oil saturation and the final oil recovery. Trapped oil saturation in the oil-wet model was higher than the water-wet model. An intermediate-wet model resulting from the nanofluid flooding gave less oil trapping compared to both the water-wet and oil-wet models.

2. Wettability alteration during nanofluid flooding was shown to be a time-dependent process. Initially, a thin oil film protected the pore walls from the nanoparticle adsorption. After a few hours, the effect of nanoparticles on wettability alteration toward intermediate-wet became more significant.

3. When silica nanoparticles were adsorbed on the solid surface, they formed a nanotexture coating on the oilwet surface. This phenomenon alters the wettability state of the surface from oil-wet to intermediate-wet, and this leads to higher oil recovery.

4. The simulation results are in good agreement with experimental results. A significant increase in oil recovery of $9 \%$ and $13 \%$ was observed for nanofluid flooding in the experimental tests and the CFD calculations, respectively.

Open Access This article is distributed under the terms of the Creative Commons Attribution 4.0 International License (http://creativeco mmons.org/licenses/by/4.0/), which permits unrestricted use, distribution, and reproduction in any medium, provided you give appropriate credit to the original author(s) and the source, provide a link to the Creative Commons license, and indicate if changes were made.

\section{Appendix: Governing equation of VOF model}

The volume fraction equations for the secondary phases and the algebraic expressions of mass and momentum equations are as follows (Fluent 2009):

Conservation of mass:

$\frac{\partial}{\partial t}(\rho)+\nabla \cdot(\rho \vec{v})=0$ where $\rho$ is density; $\vec{v}$ is velocity; and $t$ is time.

The momentum equation, shown below, is dependent on the volume fractions of all phases through the properties $\rho$ and $\mu$.

$\frac{\partial}{\partial t}(\rho \vec{v})+\nabla \cdot(\rho \vec{v} \vec{v})=-\nabla p+\nabla \cdot\left[\mu\left(\nabla \vec{v}+\nabla \vec{v}^{\mathrm{T}}\right)\right]+\rho \vec{g}+\vec{F}$

where $\vec{F}$ is force; $\mu$ is viscosity; and $\vec{g}$ is ground acceleration.

The energy equation, also shared among the phases, is shown below (Fluent 2009).

$\frac{\partial}{\partial t}(\rho E)+\nabla \cdot(\vec{v}(\rho E+p))=\nabla \cdot\left(K_{\mathrm{eff}} \nabla T\right)+S_{\mathrm{h}}$

The VOF model treats energy $E$ and temperature $T$ as mass-averaged variables:

$E=\frac{\sum_{q=1}^{n} \alpha_{q} \rho_{q} E_{q}}{\sum_{q=1}^{n} \alpha_{q} \rho_{q}}$

where $E$ is treats energy; $\rho$ is density; $K_{\text {eff }}$ is the effective thermal conductivity; $S_{\mathrm{h}}$ is volumetric source for latent heat; $E_{q}$ is treats energy for each phase; and $\alpha_{q}$ is the face value of the $q$ th volume fraction and $q$ refer to each phase.

$E_{q}$ for each phase is based on the specific heat of that phase and the shared temperature. The properties $\rho$ and $K_{\text {eff }}$ (effective thermal conductivity) are shared by the phases. The source term $S_{\mathrm{h}}$ contains contributions from radiation, as well as any other volumetric heat sources.

For time-dependent VOF calculations, the equation below is solved using an implicit time-marching scheme and Ansys Fluent's standard finite-difference interpolation scheme, and second-order upwind is used to obtain the face fluxes for all cells, including those near the interface.

$$
\begin{gathered}
\frac{\alpha_{q}^{n+1} \rho_{q}^{n+1}-\alpha_{q}^{n} \rho_{q}^{n}}{\Delta t} V+\sum_{f}\left(\rho_{q}^{n+1} U_{f}^{n+1} \alpha_{q, f}^{n+1}\right) \\
=\left[S_{a q}+\sum_{p=1}^{n}\left(\dot{m}_{p q}-\dot{m}_{q p}\right)\right] V
\end{gathered}
$$

where $n$ is the index for previous time step; $n+1$ is the index for new (current) time step; $U_{f}$ is the volume flux through the face, based on normal velocity; $\alpha_{q, f}$ is the face value of the $q$ th volume fraction, computed from the first- or second-order upwind, QUICK, modified HRIC or CICSAM schemes; $S_{a q}$ is the mass source for each phase; $V$ is the volume of cell; $\dot{m}_{p q}$ is the mass transfer from phase $p$ to phase $q$; and $\dot{m}_{q p}$ is the mass transfer from phase $q$ to phase $p$.

The properties appearing in the transport equations are determined by the presence of the component phases in each control volume. In general, for an $n$-phase system, the 
volume fraction-averaged density and viscosity take on the following form:

$$
\begin{aligned}
& \rho=\sum \alpha_{q} \rho_{q} \\
& \mu=\sum \alpha_{q} \mu_{q}
\end{aligned}
$$

\section{References}

Afsharpoor A, Ma K, Duboin A, Mateen K, Jouenne S, Cordelier P. Micro-scale experiment and CFD modeling of viscoelastic polymer; trapped oil displacement and deformation at the dead-end. In: SPE improved oil recovery symposium, Tulsa, Oklahoma, USA; 2014. https://doi.org/10.2118/169037-MS.

Anderson JD, Wendt J. Computational fluid dynamics, vol. 206. Berlin: Springer; 1995.

Dehkordi PB, Colombo LPM, Guilizzoni M, Sotgia G. CFD simulation with experimental validation of oil-water core-annular flows through Venturi and Nozzle flow meters. J Pet Sci Eng. 2017;149:540-52. https://doi.org/10.1016/j.petrol.2016.10.058.

Blunt MJ, Bijeljic B, Dong H, Gharbi O, Iglauer S, Mostaghimi P, Pentland C. Pore-scale imaging and modelling. Adv Water Resour. 2013;51:197-216. https://doi.org/10.1016/j.advwa tres.2012.03.003.

Boda D, Chan K-Y, Henderson D, Wasan DT, Nikolov AD. Structure and pressure of a hard sphere fluid in a wedge-shaped cell or meniscus. Langmuir. 1999;15(13):4311-3. https://doi. org/10.1021/la981013h.

Brackbill JU, Kothe DB, Zemach C. A continuum method for modeling surface tension. J Comput Phys. 1992;100(2):335-54. https://doi. org/10.1016/0021-9991(92)90240-Y.

Buchgraber M, Clemens T, Castanier LM, Kovscek A. A microvisual study of the displacement of viscous oil by polymer solutions. SPE Reserv Eval Eng. 2011;14(03):269-80. https://doi. org/10.2118/122400-PA.

Chareyre B, Cortis A, Catalano E, Barthélemy E. Pore-scale modeling of viscous flow and induced forces in dense sphere packings. Transp Porous Media. 2012;94(2):595-615. https://doi. org/10.1007/s11242-012-0057-2.

Chatenever A, Calhoun JC Jr. Visual examinations of fluid behavior in porous media: part I. J Pet Technol. 1952;4(06):149-56. https:// doi.org/10.2118/135-G.

Cheraghian G. An experimental study of surfactant polymer for enhanced heavy oil recovery using a glass micromodel by adding nanoclay. Pet Sci Technol. 2015;33(13-14):1410-7. https://doi. org/10.1080/10916466.2015.1062780.

Cheraghian G, Tardasti S. Improved oil recovery by the efficiency of nano-particle in imbibition mechanism. In: The 2nd EAGE international conference, KazGeo; 2012. https://doi.org/10.3997/22144609.20142967.

Conn CA, Ma K, Hirasaki GJ, Biswal SL. Visualizing oil displacement with foam in a microfluidic device with permeability contrast. Lab Chip. 2014;14(20):3968-77. https://doi.org/10.1039/C4LC0 $0620 \mathrm{H}$.

Dong M, Liu Q, Li A. Micromodel study of the displacement mechanisms of enhanced heavy oil recovery by alkaline flooding. In: Proceedings of the international symposium of the society of core analysts; 2007. p. 10-2.

Erriguible A, Bernada P, Couture F, Roques M. Simulation of convective drying of a porous medium with boundary conditions provided by CFD. Chem Eng Res Des. 2006;84(2):113-23. https:// doi.org/10.1205/cherd.05047.
Farhadi H, Riahi S, Ayatollahi S, Ahmadi H. Experimental study of nanoparticle-surfactant-stabilized $\mathrm{CO}_{2}$ foam: stability and mobility control. Chem Eng Res Des. 2016;2016(111):449-60. https:// doi.org/10.1016/j.cherd.2016.05.024.

Fluent A. 12.0/12.1 Documentation. User's guide manual. Canonsburg: Ansys Inc.; 2009.

Gahrooei HRE, Ghazanfari MH. Application of a water based nanofluid for wettability alteration of sandstone reservoir rocks to preferentially gas wetting condition. J Mol Liq. 2017;232:351-60. https ://doi.org/10.1016/j.molliq.2017.02.097.

Gharibshahi R, Jafari A, Haghtalab A, Karambeigi MS. Application of CFD to evaluate the pore morphology effect on nanofluid flooding for enhanced oil recovery. RSC Adv. 2015;5(37):28938-49. https ://doi.org/10.1039/C4RA15452E.

Hendraningrat L, Li S, Torsaeter O. Enhancing oil recovery of low-permeability Berea sandstone through optimised nanofluids concentration. In: SPE enhanced oil recovery conference, Kuala Lumpur, Malaysia; 2013. https://doi.org/10.2118/165283-MS.

Icardi M, Boccardo G, Marchisio DL, Tosco T, Sethi R. Pore-scale simulation of fluid flow and solute dispersion in three-dimensional porous media. Phys Rev E. 2014;90(1):013032. https:// doi.org/10.1103/PhysRevE.90.013032.

Jamaloei BY, Kharrat R. Analysis of microscopic displacement mechanisms of dilute surfactant flooding in oil-wet and water-wet porous media. Transp Porous Media. 2010;81(1):1. https://doi. org/10.1007/s11242-009-9382-5.

Joekar-Niasar V, van Dijke MIJ, Hassanizadeh SM. Pore-scale modeling of multiphase flow and transport: achievements and perspectives. Transp Porous Media. 2012;94(2):461-4. https://doi. org/10.1007/s11242-012-0047-4.

Ju B, Fan T, Ma M. Enhanced oil recovery by flooding with hydrophilic nanoparticles. China Particuol. 2006;4(1):41-6. https://doi. org/10.1016/S1672-2515(07)60232-2.

Kim Y, Wan J, Kneafsey TJ, Tokunaga TK. Dewetting of silica surfaces upon reactions with supercritical $\mathrm{CO}_{2}$ and brine: pore-scale studies in micromodels. Environ Sci Technol. 2012;46(7):4228-35. https://doi.org/10.1021/es204096w.

Li R, Jiang P, Gao C, Huang F, Xu R, Chen X. Experimental investigation of silica-based nanofluid enhanced oil recovery: the effect of wettability alteration. Energy Fuels. 2017;31(1):188-97. https:// doi.org/10.1021/acs.energyfuels.6b02001.

Li S, Torsæter O. An experimental investigation of EOR mechanisms for nanoparticles fluid in glass micromodel. In: The international society of core analysts annual symposium, Avignon, France; 2014. https://doi.org/10.13140/RG.2.1.4181.3604.

Liontas R, Ma K, Hirasaki GJ, Biswal SL. Neighbor-induced bubble pinch-off: novel mechanisms of in situ foam generation in microfluidic channels. Soft Matter. 2013;9(46):10971-84. https://doi. org/10.1039/C3SM51605A.

Lu T, Li Z, Zhou Y, Zhang C. Enhanced oil recovery of low-permeability cores by $\mathrm{SiO}_{2}$ nanofluid. Energy Fuels. 2017;31(5):5612-21. https://doi.org/10.1021/acs.energyfuels.7b00144.

Lv Q, Li Z, Li B, Shi D, Zhang C, Li B. Silica nanoparticles as a high-performance filtrate reducer for foam fluid in porous media. J Ind Eng Chem. 2017;45:171-81. https://doi.org/10.1016/j. jiec.2016.09.020.

Ma K, Liontas R, Conn CA, Hirasaki GJ, Biswal SL. Visualization of improved sweep with foam in heterogeneous porous media using microfluidics. Soft Matter. 2012;8(41):10669-75. https://doi. org/10.1039/C2SM25833A.

Ma K, Rivera J, Hirasaki GJ, Biswal SL. Wettability control and patterning of PDMS using UV-ozone and water immersion. J Colloid Interface Sci. 2011;363(1):371-8. https://doi.org/10.1016/j. jcis.2011.07.036.

Maghzi A, Mohebbi A, Kharrat R, Ghazanfari MH. Pore-scale monitoring of wettability alteration by silica nanoparticles during polymer 
flooding to heavy oil in a five-spot glass micromodel. Transp Porous Media. 2011;87(3):653-64. https://doi.org/10.1007/s1124 2-010-9696-3.

Miranda CR, Lara LS, Tonetto BC. Stability and mobility of functionalized silica nanoparticles for enhanced oil recovery applications. In: SPE international oilfield nanotechnology conference and exhibition, Noordwijk, The Netherlands: 2012. https://doi. org/10.2118/157033-MS.

Moon T. Nanofluid technology promises large-scale performance gains from tight reservoirs. J. Pet. Technol. 2010.

Roustaei A, Saffarzadeh S, Mohammadi M. An evaluation of modified silica nanoparticles' efficiency in enhancing oil recovery of light and intermediate oil reservoirs. Egypt J Pet. 2013;22(3):427-33. https://doi.org/10.1016/j.ejpe.2013.06.010.

Ryoo S, Rahmani AR, Yoon KY, Prodanović M, Kotsmar C, Milner TE, Huh C. Theoretical and experimental investigation of the motion of multiphase fluids containing paramagnetic nanoparticles in porous media. J Pet Sci Eng. 2012;81:129-44. https://doi. org/10.1016/j.petrol.2011.11.008.
Suleimanov BA, Ismailov FS, Veliyev EF. Nanofluid for enhanced oil recovery. J Pet Sci Eng. 2011;78(2):431-7. https://doi. org/10.1016/j.petrol.2011.06.014.

Wasan DT, Nikolov AD. Spreading of nanofluids on solids. Nature. 2003;423:156-9. https://doi.org/10.1038/nature01591.

Wendt J. Computational fluid dynamics: an introduction. Berlin: Springer; 2008.

Jamaloei BY, Babolmorad R, Kharrat R. Visualization and analysis of viscous fingering in alcohol-assisted surfactant waterflooding of heavy oil in a two-dimensional sandstone micromodel. Fuel. 2016;184:169-79. https://doi.org/10.1016/j.fuel.2016.07.016.

Yu H, He Y, Li P, Li SS, Zhang T, Rodriguez-Pin E, Huh C. Flow enhancement of water-based nanoparticle dispersion through microscale sedimentary rocks. Sci Rep. 2015;5:8702. https://doi. org/10.1038/srep08702.

Zhang H, Ramakrishnan TS, Nikolov A, Wasan D. Enhanced oil displacement by nanofluid's structural disjoining pressure in model fractured porous media. J Colloid Interface Sci. 2018;511:48-56. https://doi.org/10.1016/j.jcis.2017.09.067. 